\title{
On uniqueness and structure of renormalized solutions to integro-differential equations with general measure data
}

\author{
Tomasz Klimsiak(
}

\begin{abstract}
We propose a new definition of renormalized solution to linear equation with self-adjoint operator generating a Markov semigroup and bounded Borel measure on the right-hand side. We give a uniqueness result and study the structure of solutions to truncated equations.

Mathematics Subject Classification. Primary 35R06; Secondary 35R05, 45K05, 47G20, 35D99.

Keywords. Renormalized solution, Dirichlet form, Measure data, Markov semigroup, Markov process, Green function.
\end{abstract}

\section{Introduction}

In this paper, $E$ is a locally compact separable metric space and $m$ is a Radon measure on $E$ with full support. Let $(A, D(A))$ be a non-positive definite selfadjoint operator on $L^{2}(E ; m)$ associated with some Dirichlet form $(\mathcal{E}, D(\mathcal{E}))$ on $L^{2}(E ; m)$. The main goal of the present paper is to give a new definition of renormalized solution to the linear equation

$$
-A u=\mu
$$

with general (possibly nonsmooth in the Dirichlet forms theory sense) bounded Borel measure $\mu$ on $E$. It is known that such a measure admits unique decomposition

$$
\mu=\mu_{d}+\mu_{c}
$$

into the absolutely continuous, with respect to the capacity Cap generated by $\left(\mathcal{E}, D(\mathcal{E})\right.$ ), part $\mu_{d}$ (so-called diffuse part or smooth part of $\mu$ ) and the orthogonal, with respect to Cap, part $\mu_{c}$ (so-called concentrated part). The problem of right definition to (1.1) is rather subtle if we require that the solution $u$ be unique. 
In the paper, we assume that the resolvent $\left(R_{\alpha}\right)_{\alpha>0}$ generated by $A$ is Fellerian, i.e. $R_{\alpha}\left(C_{b}(E)\right) \subset C_{b}(E)$ for some (and hence for all) $\alpha>0$, and there exists a Green function $G$ for $A$ (see Sect. 2.2).

Our new definition reads as follows: $u \in \mathcal{B}(E)$ is a renormalized solution to $(1.1)$ if

(i) $T_{k}(u):=(u \wedge k) \vee(-k) \in D_{e}(\mathcal{E}), k \geq 0$, where $D_{e}(\mathcal{E})$ is the extended Dirichlet space, i.e. an extension of $D(\mathcal{E})$ such that $D_{e}(\mathcal{E})$ with inner product $\mathcal{E}$ is a Hilbert space.

(ii) There exists a family of bounded smooth measures $\left(\nu_{k}\right)_{k \geq 0}$ on $E$ such that

$$
\mathcal{E}\left(T_{k}(u), \eta\right)=\left\langle\mu_{d}, \eta\right\rangle+\left\langle\nu_{k}, \eta\right\rangle, \quad \eta \in D_{e}(\mathcal{E}) \cap \mathcal{B}_{b}(E), \quad k \geq 0,
$$

(iii) $\nu_{k} \rightarrow \mu_{c}$ in the narrow topology, i.e. for every $\eta \in C_{b}(E)$,

$$
\lim _{k \rightarrow \infty} \int_{E} \eta d \nu_{k}=\int_{E} \eta d \mu_{c}
$$

A similar definition of a solution to (1.1), also guaranteeing uniqueness, was introduced recently in my joint paper with Rozkosz [10]. In that paper by a solution we mean $u \in \mathcal{B}(E)$ satisfying (i) and (ii), and the following condition (iii') $\lim _{k \rightarrow \infty} \int_{E} G(x, y) \nu_{k}(d y)=\int_{E} G(x, y) \mu_{c}(d y)$ for $m$-a.e. $x \in E$.

Condition (iii) is much simpler than (iii') because it does not involves the notion of the Green function. One of the main results of the present paper says that (i)-(iii) still ensure uniqueness for solutions to (1.1). In the second part of the paper we show interesting properties of the family $\left(\nu_{k}\right)_{k \geq 0}$ : a structure theorem, so-called reconstruction property and the narrow convergence of variations.

The above definition (i)-(iii) is a counterpart to the definition introduced by Dal Maso et al. [3] for equations with local nonlinear operators of LerayLions type of the form

$$
A(u)=\operatorname{div}(a(\cdot, \nabla u)) .
$$

For such operators, $\mathcal{E}$ appearing in (i), (ii) is given by

$$
\mathcal{E}(u, v):=\int_{E} a(\cdot, \nabla u) \nabla v d m,
$$

and the domain of $\mathcal{E}$ is the natural energy space in which $\mathcal{E}(u, u)$ is finite. As a matter of fact, this modified definition (i)-(iii) is one of the four equivalent definitions of renormalized solutions considered in [3].

The concept of renormalized solutions was a crucial step in the development of the theory of elliptic and parabolic equations with (nonlinear) local operators and measure data since it gives partial uniqueness results. The complete uniqueness result in nonlinear case is still an open problem.

It is worth noting here that among the definitions considered in [3] the definition presented above have some remarkable feature. Namely, in condition (ii) the term $\mathcal{E}\left(T_{k}(u), \eta\right)$ is well defined since both $T_{k}(u)$ and $\eta$ are in the domain of $\mathcal{E}$. In the other definitions considered in [3], a different variational formulas [counterparts to (ii)] are considered. In these formulas the 
term $\mathcal{E}(u, \eta)$ always appears which of course requires an extension of the form $(\mathcal{E}, D(\mathcal{E}))$ in such a way that $\mathcal{E}(u, \eta)$ makes sense for rich enough class of test functions $\eta$. All the used extensions of $\mathcal{E}$ in [3] are based on the property

$$
\mathcal{E}\left(u, T_{k}(u)\right)=\mathcal{E}\left(T_{k}(u), T_{k}(u)\right),
$$

which is true only for the forms associated with local operators. For that reason only definition of type (i)-(iii) can be directly adopted to the nonlocal case. In case $\mathcal{E}$ is nonlocal of the form

$$
\mathcal{E}(u, v)=\int_{E \times E}(u(x)-u(y))(v(x)-v(y)) J(d x, d y)
$$

for some symmetric positive measure $J$ on $E \times E \backslash d$, Alibaud et al. [1] proposed the following extension of $\mathcal{E}$ : for $h \in C_{c}^{1}(\mathbb{R})$

$$
\begin{aligned}
\mathcal{E}(u, h(u) \eta):= & \int_{E \times E}(u(x)-u(y))(h(u)(x)-h(u)(y)) \frac{\eta(x)+\eta(y)}{2} J(d x, d y) \\
& +\int_{E \times E}(u(x)-u(y))(\eta(x)-\eta(y)) \frac{h(u)(x)+h(u)(y)}{2} J(d x, d y)
\end{aligned}
$$

for bounded $\eta$ and $u$ such that

$$
\int_{E \times E}(u(x)-u(y))\left(T_{k}(u)(x)-T_{k}(u)(y)\right) J(d x, d y)<\infty, \quad k \geq 0 .
$$

A careful analysis shows that thanks to (1.5) and regularity of $h$ both integrals in (1.4) are well defined. However, the crucial assumption that $h$ has compact support makes this approach applicable only to equations with smooth (diffuse) measure data. In [1], imitating one of the definition considered in [3], the authors introduced the following definition of a solution to (1.1) with $A$ generated by (1.3), $E=\mathbb{R}^{d}$ and smooth measure data (in fact for $\mu \in L^{1}\left(\mathbb{R}^{d}\right.$ ) but it naturally extends to smooth measure data): a measurable function $u$ satisfying (1.5) is a renormalized solution to (1.1) if

$$
\mathcal{E}(u, h(u) \eta)=\langle\mu, h(u) \eta\rangle, \quad \eta \in C^{\infty}\left(\mathbb{R}^{d}\right), h \in C_{c}^{1}\left(\mathbb{R}^{d}\right)
$$

and

$$
\begin{gathered}
\lim _{k \rightarrow \infty} \int_{E \times E}(u(x)-u(y))\left\{\left(T_{k+1}(u)-T_{k}(u)\right)(x)\right. \\
\left.-\left(T_{k+1}(u)-T_{k}(u)\right)(y)\right\} J(d x, d y)=0 .
\end{gathered}
$$

We extend this definition to general forms $\mathcal{E}$ considered here (and smooth measure data) and show that if $u$ is a renormalized solution to (1.1) in the sense of definition (i)-(iii), then $u$ is a renormalized solution to (1.1) is the sense of (1.6) and (1.7).

In order to get existence and uniqueness result for solutions to (1.1) with general measure data, we propose definition (i)-(iii) which seems to be the more suitable formulation of the definition of renormalized solution to (1.1) since it is applicable not only to general measure data but also to wide class of operators associated with local and non-local Dirichlet forms. 
We prove that for bounded Borel measure $\mu$ there exists a unique renormalized solution to (1.1). Moreover, if $u$ is renormalized solution to (1.1) then

$$
u(x)=\int_{E} G(x, y) \mu(d y) \quad m \text {-a.e. } x \in E,
$$

and even stronger convergence of $\left\{\nu_{k}\right\}$ holds. Namely

$$
\nu_{k}^{+} \rightarrow \mu_{c}^{+}, \quad \nu_{k}^{-} \rightarrow \mu_{c}^{-} \quad \text { narrowly. }
$$

From this it follows in particular that $u$ is a renormalized solution to (1.1) in the sense of (i)-(iii) if and only if $u$ is a duality solution to (1.1) in the sense of Stampacchia. The notion of duality solutions for linear equations with uniformly elliptic divergence form operators and general measure data was introduced by Stampacchia [16]. His approach was adapted to fractional Laplacian in $[5,13]$. The general formulation for operators $A$ generated by Markov semigroups was introduced in [6] (see also [7] for the case of smooth measure data).

In the second part of the paper, we give a complete characterization of the family $\left(\nu_{k}\right)_{k \geq 0}$. Recall that each regular symmetric Dirichlet form $(\mathcal{E}, D(\mathcal{E}))$ admits the following (unique) Beurling-Deny decomposition

$$
\begin{aligned}
& \mathcal{E}(u, v)=\mathcal{E}^{(c)}(u, v)+\int_{E \times E}(u(x)-u(y))(v(x)-v(y)) J(d x, d y) \\
& \quad+\int_{E} v(x) u(x) \kappa(d x) .
\end{aligned}
$$

Here $\mathcal{E}^{(c)}$ is the local part of $\mathcal{E}, J$ is a symmetric positive Radon measure outside the diagonal $d$ of $E \times E$ and $\kappa$ is a smooth Radon measure on $E$, called the killing measure. We show that

$$
\nu_{k}=-\mathbf{1}_{\{u \geq k, u<-k\}} \cdot \mu_{d}+\frac{1}{2} l_{k}(u)-\frac{1}{2} l_{-k}(u)+\frac{1}{2} j_{k}(u)-\frac{1}{2} j_{-k}(u)
$$

with

$$
\begin{aligned}
j_{k}(u)(d x)= & 2 \int_{E}(|u(y)-k|-|u(x)-k|-\operatorname{sign}(u(x)-k)(u(y)-u(x))) J(d x, d y) \\
& +\left(\mathbf{1}_{\{u(x)>k\}}(|k|+k)+\mathbf{1}_{\{u(x) \leq k\}}(|k|-k)\right) \kappa(d x) .
\end{aligned}
$$

and $\left\{l_{k}(u), k \in \mathbb{Z}\right\}$ characterized as follows

$$
\int_{\mathbb{R}}\left\langle l_{k}(u), \eta\right\rangle \psi(k) d k=\left\langle\mu_{\langle u\rangle}^{c}, \psi(u) \eta\right\rangle, \quad \psi, \eta \in \mathcal{B}^{+}(E),
$$

where $\mu_{\langle u\rangle}^{c}$ is a positive smooth Radon measure on $E$ given by

$$
\left\langle\mu_{\langle u\rangle}^{c}, \eta\right\rangle=\lim _{k \rightarrow \infty} 2 \mathcal{E}^{(c)}\left(T_{k}(u) \eta, T_{k}(u)\right)-\mathcal{E}^{(c)}\left(T_{k}(u)^{2}, \eta\right), \quad \eta \in \mathcal{B}_{b}(E) \cap D(\mathcal{E}) .
$$

From (1.10) it follows in particular that if $\mathcal{E}$ is local (i.e. $J \equiv 0$ ), then

$$
\frac{1}{c_{n}-b_{n}} \int_{\left\{b_{n} \leq u \leq c_{n}\right\}} \eta d \mu_{\langle u\rangle}^{c} \rightarrow\left\langle\mu_{c}^{+}, \eta\right\rangle,
$$




$$
\frac{1}{c_{n}-b_{n}} \int_{\left\{-c_{n} \leq u \leq-b_{n}\right\}} \eta d \mu_{\langle u\rangle}^{c} \rightarrow\left\langle\mu_{c}^{-}, \eta\right\rangle,
$$

for all sequences $\left\{b_{n}\right\},\left\{c_{n}\right\}$ of positive numbers such that $b_{n}<c_{n}, n \geq 1$ and $b_{n}, c_{n} \rightarrow \infty$ as $n \rightarrow \infty$ (the so-called reconstruction property). Observe also that $j_{k}(u)$ may be concentrated on the whole $E$. Hence, contrary to the local case, the measures $\nu_{k}$ need not be concentrated on the set $\{|u|=k\}$.

In the present paper we focus our attention on linear equation (1.1). However, our results also apply to semilinear equations of the form

$$
-A u=f(\cdot, u)+\mu
$$

with $f$ being a measurable function on $E \times \mathbb{R}$. By a renormalized solution to (1.12) we mean a measurable function $u$ on $E$ such that $f(\cdot, u) \in L^{1}(E ; m)$ and (i)-(iii) hold when we replace $\mu_{d}$ by $f(\cdot, u)+\mu_{d}$ in condition (ii) (since $(f(\cdot, u)+$ $\left.\mu)_{d}=f(\cdot, u)+\mu_{d}\right)$. From our results it follows that if $f$ is nonincreasing with respect to the second variable, then there exists at most one renormalized solution to (1.12).

\section{Notation and standing assumptions}

In the paper, $E$ is a locally compact separable metric space and $\partial$ is a onepoint compactification of $E$. If $E$ is already compact, the $\partial$ is an isolated point. We adopt the convention that each function $f$ on $E$ is extended to $E \cup\{\partial\}$ by setting $f(\partial)=0$.

We denote by $\mathcal{B}(E)$ the set of Borel measurable functions on $E$. $\mathcal{B}_{b}(E)$, $\mathcal{B}^{+}(E)$ are the subsets of $\mathcal{B}(E)$ consisting of bounded and positive functions, respectively. We denote by $\mathcal{M}(E)$ the set of Borel measures on $E$, and by $\mathcal{M}^{+}(E)$ the subset of $\mathcal{M}(E)$ consisting of positive measures. For given $\eta \in$ $\mathcal{B}^{+}(E)$ and $\mu \in \mathcal{M}^{+}(E)$ we set

$$
\langle\mu, \eta\rangle=\int_{E} \eta d \mu,
$$

whenever the integral exists. For given $u \in \mathcal{B}^{+}(E)$ and $\mu \in \mathcal{M}^{+}(E)$, we denote by $u \cdot \mu$ the measure on $E$ defined by

$$
\langle u \cdot \mu, \eta\rangle=\langle\mu, u \eta\rangle, \quad \eta \in \mathcal{B}^{+}(E) .
$$

\subsection{Dirichlet forms and potential theory}

In the whole paper, $(\mathcal{E}, D(\mathcal{E}))$ is a symmetric Dirichlet form on $L^{2}(E ; m)$ which is regular, that is the set $C_{c}(E) \cap D(\mathcal{E})$ is dense in $C_{c}(E)$ with uniform norm, and in $D(\mathcal{E})$ with $\mathcal{E}_{1}$-norm, where for $\alpha>0, \mathcal{E}_{\alpha}(u, v)=\mathcal{E}(u, v)+\alpha(u, v)$, $u, v \in D(\mathcal{E})$. We also assume that it is transient, that is there exists a strictly positive function $g$ on $E$ such that

$$
\int_{E}|u| g d m \leq c \sqrt{\mathcal{E}(u, u)}, \quad u \in D(\mathcal{E}) .
$$

Therefore, by $\left[4\right.$, Theorem 1.5.3], there exists an extension $D_{e}(\mathcal{E}) \subset L^{1}(E, g$. $m$ ) of $D(\mathcal{E})$ such that $\left(\mathcal{E}, D_{e}(\mathcal{E})\right)$ is a Hilbert space. By [4, Theorem 1.3.1], 
there exists a unique self-adjoint non-positive definite operator $(A, D(A))$ on $L^{2}(E ; m)$ such that

$$
D(A) \subset D(\mathcal{E}), \quad \mathcal{E}(u, v)=(-A u, v), \quad u \in D(A), v \in L^{2}(E ; m) .
$$

We denote by $\left(T_{t}\right)_{t \geq 0}$ the semigroup of contractions on $L^{2}(E ; m)$ generated by $(A, D(A))$ and by Cap the capacity on $E$ defined as follows: for and open $U \subset E$,

$$
\operatorname{Cap}(U)=\inf \left\{\mathcal{E}(u, u): u \in D(\mathcal{E}), u \geq \mathbf{1}_{U} m \text {-a.e. }\right\},
$$

and for an arbitrary $B \subset E$,

$$
\operatorname{Cap}(B)=\inf \{\operatorname{Cap}(U): B \subset U, U \subset E \text { is open }\} .
$$

We say that a property $P$ holds quasi everywhere (q.e. in abbreviation) if it holds outside a set $N$ with $\operatorname{Cap}(N)=0$. We say that a measurable function $u$ on $E$ is quasi-continuous if for every $\varepsilon>0$ there exists a closed set $F_{\varepsilon} \subset E$ such that $\operatorname{Cap}\left(E \backslash F_{\varepsilon}\right)<\varepsilon$ and $u_{\mid F_{\varepsilon}}$ is continuous. By [4, Theorem 2.1.7], every function $u \in D_{e}(\mathcal{E})$ has an $m$-version $\tilde{u}$ which is quasi-continuous.

For a Borel measure on $E,|\mu|$ stands for its total variation. We say that a Borel measure $\mu$ on $E$ is smooth if $|\mu|(B)=0$ for every Borel set $B \subset E$ such that $\operatorname{Cap}(B)=0$, and there exists a strictly positive quasi-continuous function $\eta$ on $E$ such that $\langle|\mu|, \eta\rangle<\infty$. The set of all positive smooth measures on $E$ will be denoted by $S$. We denote by $\mathcal{M}_{0, b}(E)$ the set of bounded smooth measures, and by $\mathcal{M}_{0, b}^{+}(E)$ the set of positive bounded smooth measures. Each $\mu \in \mathcal{M}(E)$ admits unique decomposition of the form (1.2), where $\mu_{d}$ is a smooth measure and $\mu_{c}$ is concentrated on the set $B \subset E$ such that $\operatorname{Cap}(B)=0$.

\subsection{Probabilistic potential theory}

By $[4$, Section 7$]$, there exists a Hunt process $\mathbb{X}=\left\{\left(X_{t}\right)_{t \geq 0},\left(P_{x}\right)_{x \in E \cup \partial}, \mathbb{F}:=\right.$ $\left.\left(\mathcal{F}_{t}\right)_{t \geq 0}\right\}$ with life time $\zeta$ associated with the Dirichlet form $(\mathcal{E}, D(\mathcal{E}))$ in the sense that for every $\eta \in L^{2}(E ; m) \cap \mathcal{B}^{+}(E)$,

$$
T_{t} f(x)=E_{x} f\left(X_{t}\right), \quad m \text {-a.e. }
$$

In the paper, we assume that $\mathbb{X}$ satisfies absolute continuity condition, i.e. there exists a positive Borel function $p$ on $\mathbb{R}^{+} \times E \times E$ such that for all $x \in E$, $t>0$ and $f \in \mathcal{B}^{+}(E)$,

$$
E_{x} f\left(X_{t}\right)=\int_{E} f(y) p(t, x, y) m(d y) .
$$

We denote by $\left(P_{t}\right)_{t \geq 0}$ (resp. $\left.\left(R_{\alpha}\right)_{\alpha>0}\right)$ the semigroup (resp. resolvent) associated with $\mathbb{X}$. Recall that for all $t, \alpha \geq 0$ and $f \in \mathcal{B}^{+}(E)$,

$$
P_{t} f(x)=E_{x} f\left(X_{t}\right), \quad R_{\alpha} f(x)=E_{x} \int_{0}^{\zeta} e^{-\alpha t} f\left(X_{t}\right) d t, \quad x \in E,
$$

We set $R:=R_{0}$. For $\mu \in \mathcal{M}^{+}(E)$, we set

$$
R \mu(x)=\int_{E} G(x, y) \mu(d y), \quad x \in E .
$$


Here $G$ is the Green function defined by

$$
G(x, y)=\int_{0}^{\infty} p(t, x, y) d t, \quad x, y \in E .
$$

In the sequel, we write that some property $P$ holds q.a.s. (resp. a.s.) if it holds outside a set $B \in \mathcal{F}_{\infty}$ such that $P_{x}(B)=0$ for q.e. $x \in E$ (resp. for every $x \in E$ ).

Recall that $u \in \mathcal{B}^{+}(E)$ is called excessive if

$$
\sup _{t>0} P_{t} u(x)=u(x), \quad x \in E .
$$

The set of all excessive functions will be denoted by Exc. It is well known that for any $\eta, \psi \in$ Exc the mapping

$$
\mathbb{R}^{+} \ni t \mapsto \frac{1}{t}\left\langle\psi, \eta-P_{t} \eta\right\rangle
$$

is nonincreasing. Moreover, if $\psi=R \mu$ for some positive Borel measure $\mu$ and $\psi<\infty$ m-a.e., then

$$
\sup _{t>0} \frac{1}{t}\left\langle\psi, \eta-P_{t} \eta\right\rangle=\lim _{t \rightarrow 0^{+}} \frac{1}{t}\left\langle\psi, \eta-P_{t} \eta\right\rangle=\langle\mu, \eta\rangle .
$$

Let $\mathbf{A}_{c}^{+}$denote the set of positive continuous additive functionals of $\mathbb{X}$ (see [4, Section 5.1]). It is well known that there exists an isomorphism (the so-called Revuz duality)

$$
\mathcal{R}: \mathbf{A}_{c}^{+}-\mathbf{A}_{c}^{+} \rightarrow S-S
$$

defined as follows: for every $A \in \mathbf{A}_{c}^{+}$and every continuous positive $\eta$ on $E$,

$$
\langle\mathcal{R}(A), \eta\rangle=\lim _{t \rightarrow 0^{+}} \frac{1}{t} E_{m} \int_{0}^{t} \eta\left(X_{r}\right) d A_{r} .
$$

By [4, Theorem 5.1.3], $t \mapsto \frac{1}{t} E_{m} \int_{0}^{t} \eta\left(X_{r}\right) d A_{r}$ is nonincreasing for any $\eta \in E x c$ and $A \in \mathbf{A}_{c}^{+}$, and the above equality also holds for $\eta \in E x c$. Moreover, if $\eta$ is excessive or is a positive continuous function $\eta$ on $E$, then

$$
\lim _{t \rightarrow 0^{+}} \frac{1}{t} E_{m} \int_{0}^{t} \eta\left(X_{r}\right) d A_{r}=\lim _{t \rightarrow 0^{+}} \frac{1}{t} E_{\eta \cdot m} \int_{0}^{t} d A_{r} .
$$

In the sequel, for given $\mu \in S$ we set $A^{\mu}:=\mathcal{R}^{-1}(\mu)$. By [4, Theorem 5.1.3], for all $\eta \in \mathcal{B}^{+}(E)$ and $\mu \in S$,

$$
E_{x} \int_{0}^{\zeta} \eta\left(X_{t}\right) d A_{t}^{\mu}=\int_{E} \eta(y) G(x, y) \mu(d y), \quad x \in E .
$$

For a given càdlàg special semimartingale $Y$ and $k \in \mathbb{R}$, we denote by $L^{k}(Y)$ the local time of $Y$ at $k$ (see [15, p. 212]). We also put

$$
J_{t}^{k}(Y)=\sum_{s \leq t}\left(\left|Y_{s}-k\right|-\left|Y_{s-}-k\right|-\operatorname{sign}\left(Y_{s-}-k\right) \Delta Y_{s}\right),
$$

where $\Delta Y_{t}=Y_{t}-Y_{t-}, Y_{t-}=\lim _{s \rightarrow t^{-}} Y_{s}$ and $\operatorname{sign}(x)=1$ if $x>0$ and $\operatorname{sign}(x)=-1$ if $x \leq 0$. Since $x \mapsto|x-k|$ is a convex function, $J^{k}$ is an 
increasing process. By the Tanaka-Meyer formula (see [15, Theorem 70, p. 214]) for every convex function $\varphi: \mathbb{R} \rightarrow \mathbb{R}$,

$$
\varphi\left(Y_{t}\right)=\varphi\left(Y_{0}\right)+\int_{0}^{t} \varphi^{\prime}\left(Y_{s-}\right) d Y_{s}+\frac{1}{2} \int_{\mathbb{R}} L_{t}^{k}(Y) \mu(d k)+\frac{1}{2} \int_{\mathbb{R}} J_{t}^{k}(Y) \mu(d k),
$$

where $\varphi^{\prime}$ is the left derivative of $\varphi$ and $\mu=\left(\varphi^{\prime}\right)^{\prime}$ with second derivative taken in distributional sense. Since $Y$ is a special semimartingale, there exists a process ${ }^{p} J^{k}(Y)$ which is the dual predictable projection of $J^{k}(Y)$. In case $Y=u(X)$ q.a.s. it is easy to observe that $L^{k}(u(X))$ and $J^{k}(u(X))$ are positive additive functionals. By the definition of local time, $L^{k}(u(X))$ is continuous, and since $\mathbb{X}$ is a Hunt process, ${ }^{p} J^{k}(u(X))$ is continuous too (and it is still a positive additive functional, see [4, Theorem A.3.16]). We set

$$
l_{k}(u)=\mathcal{R}\left(L^{k}(u(X))\right), \quad j_{k}(u)=\mathcal{R}\left({ }^{p} J^{k}(u(X))\right), \quad \lambda_{k}(u)=l_{k}(u)+j_{k}(u) .
$$

By [15, Theorem IV.69], the measure $l_{k}(u)$ is concentrated on the set $\{u=k\}$.

\section{Integral, duality, probabilistic and very weak solutions}

Definition 3.1. Let $\mu \in \mathcal{M}_{b}(E)$. We say that $u \in \mathcal{B}(E)$ is an integral solution to $(1.1)$ if

$$
u(x)=\int_{E} G(x, y) \mu(d y) \quad m \text {-a.e. } x \in E .
$$

Remark 3.2. Let $u$ be an integral solution to (1.1). Define

$$
N=\left\{x \in E: \int_{E} G(x, y)|\mu|(d y)=\infty\right\} .
$$

By [6, Proposition 3.2], $\operatorname{Cap}(N)=0$, and by [6, Theorem 3.7], $\tilde{u}$ defined as

$$
\tilde{u}(x)=0, \quad x \in N, \quad \tilde{u}(x)=\int_{E} G(x, y) \mu(d y), \quad x \in E \backslash N,
$$

is a quasi-continuous $m$-version of $u$. Therefore we may assume that any integral solution $u$ to (1.1) is quasi-continuous and (3.1) is satisfied for q.e. $x \in E$.

The next definition introduced in [6] is a generalization, to the class of operators considered in the present paper, of Stampacchia's definition introduced in [16] in case $A$ is a uniformly elliptic diffusion operator in divergence form. In case $A$ is a fractional Laplacian, duality solutions were considered in [5] (on $\mathbb{R}^{d}$ ) and in [13] (on bounded domains in $\mathbb{R}^{d}$ ).

Definition 3.3. Let $\mu \in \mathcal{M}_{b}(E)$. We say that $u \in \mathcal{B}(E)$ is a duality solution to (1.1) if for every $\eta \in \mathcal{B}(E)$ such that $R|\eta|$ is bounded

$$
\langle u, \eta\rangle=\langle\mu, R \eta\rangle .
$$


It is worth noting here that in case $\mu$ is a smooth measure it is possible to define duality solutions for general operators corresponding to transient regular Dirichlet forms, i.e. without the additional assumption that there exists the Green function for $A$ (see $[7])$.

The following definition of probabilistic solution to (1.1) was introduced in [6]. To formulate it, we first recall that $M$ is called a local martingale additive functional (local MAF for short) of $\mathbb{X}$ if $M$ is an additive functional of $\mathbb{X}$ and $M$ is a local martingale under the measure $P_{x}$ for q.e. $x \in E$ (see [6] for details).

Definition 3.4. Let $\mu \in \mathcal{M}_{b}(E)$. We say that $u \in \mathcal{B}(E)$ is a probabilistic solution to (1.1) if

(i) there exists a local martingale additive functional $M$ of $\mathbb{X}$ such that

$$
u(x)=u\left(X_{t}\right)+\int_{0}^{t} d A_{r}^{\mu_{d}}-\int_{0}^{t} d M_{r}, \quad t \in[0, \zeta), \quad \text { q.a.s. }
$$

(ii) for every sequence $\left\{\tau_{k}\right\}$ of $\mathbb{F}$-stopping times such that $E_{x} \sup _{t \leq \tau_{k}}\left|u\left(X_{t}\right)\right|<$ $\infty$ for q.e. $x \in E$ and $\tau_{k} \nearrow \zeta$ q.a.s. we have

$$
E_{x}\left(u\left(X_{\tau_{k}}\right)\right) \rightarrow R \mu_{c}(x) \text { q.e. } x \in E .
$$

Any sequence $\left\{\tau_{k}\right\}$ of $\mathbb{F}$-stopping times such that $E_{x} \sup _{t \leq \tau_{k}}\left|u\left(X_{t}\right)\right|<\infty$ for q.e. $x \in E$ and $\tau_{k} \nearrow \zeta$ q.a.s. is called the reducing sequence for $u$.

Remark 3.5. From [6, Proposition 3.12] it follows that any probabilistic solution to (1.1) is quasi-continuous.

By [6, Propositions 3.12, 4.12] we have the following result.

Proposition 3.6. Let $\mu \in \mathcal{M}_{b}(E)$.

(i) If $u$ is a probabilistic solution to (1.1), then $u$ is an integral solution to (1.1).

(ii) If $u$ is an integral solution to (1.1), then $\tilde{u}$ defined by (3.2) is a probabilistic solution to (1.1).

(iii) $u$ is an integral solution to (1.1) iff it is a duality solution to (1.1).

From now on, we always consider quasi-continuous versions of solutions to (1.1) (no matter which one of the definition we consider).

Proposition 3.7. Let $\mu \in \mathcal{M}_{b}(E)$ and $u$ be an integral solution to (1.1). Then $\lambda_{k}(u) \in \mathcal{M}_{0, b}(E)$ for every $k \in \mathbb{R}$. Moreover,

$$
-A u^{+}=\mathbf{1}_{\{u>0\}} \cdot \mu_{d}-\frac{1}{2} \lambda_{0}(u)+\mu_{c}^{+},
$$

and for every $k>0$,

$$
\begin{aligned}
& -A\left(u^{+} \wedge k\right)=\mathbf{1}_{\{0<u \leq k\}} \cdot \mu_{d}+\frac{1}{2} \lambda_{k}(u)-\frac{1}{2} \lambda_{0}(u), \\
& -A\left(u^{-} \wedge k\right)=-\mathbf{1}_{\{-k<u \leq 0\}} \cdot \mu_{d}+\frac{1}{2} \lambda_{-k}(u)-\frac{1}{2} \lambda_{0}(u) .
\end{aligned}
$$


Proof. By Proposition 3.6 and the definition of a probabilistic solution to (1.1),

$$
u\left(X_{t}\right)=u\left(X_{\tau_{n}}\right)+\int_{t}^{\tau_{n}} d A_{r}^{\mu_{d}}-\int_{t}^{\tau_{n}} d M_{r}, \quad t \in\left[0, \tau_{n}\right], \text { q.a.s. },
$$

for some local MAF $M$ of $\mathbb{X}$ and reducing sequence $\left\{\tau_{n}\right\}$ for $u$. By the TanakaMeyer formula,

$$
\begin{aligned}
u^{+}\left(X_{t}\right)= & u^{+}\left(X_{\tau_{n}}\right)+\int_{t}^{\tau_{n}} \mathbf{1}_{\left\{u\left(X_{r-}\right)>0\right\}} d A_{r}^{\mu_{d}}-\frac{1}{2} \int_{t}^{\tau_{n}} d L_{r}^{0}(u(X)) \\
& -\frac{1}{2} \int_{t}^{\tau_{n}} d J_{r}^{0}(u(X))-\int_{t}^{\tau_{n}} \mathbf{1}_{\left\{u\left(X_{r-}\right)>0\right\}} d M_{r}, \quad t \in\left[0, \tau_{n}\right] \text {, q.a.s. }
\end{aligned}
$$

Hence

$$
\begin{aligned}
u^{+}\left(X_{t}\right)= & u^{+}\left(X_{\tau_{n}}\right)+\int_{t}^{\tau_{n}} \mathbf{1}_{\left\{u\left(X_{r-}\right)>0\right\}} d A_{r}^{\mu_{d}}-\frac{1}{2} \int_{t}^{\tau_{n}} d L_{r}^{0}(u(X)) \\
& -\frac{1}{2} \int_{t}^{\tau_{n}} d\left({ }^{p}\left[J_{r}^{0}(u(X))\right]\right)-\int_{t}^{\tau_{n}} d N_{r}, \quad t \in\left[0, \tau_{n}\right] \text {, q.a.s. }
\end{aligned}
$$

for some local MAF $N$ of $\mathbb{X}$. Taking the expectation of both sides of the above equation and then letting $n \rightarrow \infty$ and using (2.2) and [6, Theorem 6.3] we obtain

$$
u^{+}(x)=R\left(\mathbf{1}_{\{u>0\}} \cdot \mu_{d}\right)(x)-\frac{1}{2} R\left(\lambda_{0}(u)\right)(x)+R \mu_{c}^{+}(x), \quad \text { q.e. } x \in E .
$$

This implies (3.3). Furthermore, (3.3) when combined with [6, Lemma 4.6] implies that $\lambda_{0}(u) \in \mathcal{M}_{b}(E)$. Applying the Tanaka-Meyer formula to (3.6) (with the function $\varphi(x)=x^{+} \wedge k$ ) yields

$$
\begin{aligned}
\left(u^{+} \wedge k\right)\left(X_{t}\right)= & \left(u^{+} \wedge k\right)\left(X_{\tau_{n}}\right)+\int_{t}^{\tau_{n}} \mathbf{1}_{\left\{0<u\left(X_{r-}\right) \leq k\right\}} d A_{r}^{\mu_{d}}-\frac{1}{2} \int_{t}^{\tau_{n}} d L_{r}^{0}(u(X)) \\
& -\frac{1}{2} \int_{t}^{\tau_{n}} d\left({ }^{p}\left[J_{r}^{0}(u(X))\right]\right)+\frac{1}{2} \int_{t}^{\tau_{n}} d L_{r}^{k}(u(X)) \\
& +\frac{1}{2} \int_{t}^{\tau_{n}} d\left({ }^{p}\left[J_{r}^{k}(u(X))\right]\right)-\int_{t}^{\tau_{n}} d N_{r}, \quad t \in\left[0, \tau_{n}\right], \text { q.a.s. }
\end{aligned}
$$

for some MAF $N$ of $\mathbb{X}$. Since $u^{+} \wedge k$ is bounded, $E_{x}\left(u^{+} \wedge k\right)\left(X_{\tau_{n}}\right) \rightarrow 0$ for q.e. $x \in E$. Therefore taking the expectation of both sides of the above equation and applying (2.2) yields

$$
\left(u^{+} \wedge k\right)(x)=R\left(\mathbf{1}_{\{0<u \leq k\}} \cdot \mu_{d}\right)(x)-\frac{1}{2} R\left(\lambda_{0}(u)\right)(x)+\frac{1}{2} R\left(\lambda_{k}(u)\right)(x)
$$

for q.e. $x \in E$, which shows (3.4). Since $u^{+} \wedge k \leq u^{+}$, we have

$$
\begin{aligned}
& R\left(\mathbf{1}_{\{0<u \leq k\}} \cdot \mu_{d}\right)-\frac{1}{2} R\left(\lambda_{0}(u)\right)+\frac{1}{2} R\left(\lambda_{k}(u)\right) \\
& \quad \leq R\left(\mathbf{1}_{\{u>0\}} \cdot \mu_{d}\right)-\frac{1}{2} R\left(\lambda_{0}(u)\right)+R \mu_{c}^{+} \text {q.e. }
\end{aligned}
$$

By this and [6, Lemma 4.6] again, $\lambda_{k}(u) \in \mathcal{M}_{b}(E)$ for $k>0$. Using the same argument, but with the function $\varphi(x)=x^{-} \wedge k$, we show that $\lambda_{k}(u) \in \mathcal{M}_{b}(E)$ for $k<0$ and (3.5) is satisfied. 
Corollary 3.8. Let $u$ be an integral solution to (1.1). Then

$$
\nu_{k}=-\mathbf{1}_{\{u>k, u \leq-k\}} \mu_{d}+\frac{1}{2}\left(\lambda_{k}(u)-\lambda_{-k}(u)\right), \quad k>0 .
$$

Proof. Follows from Proposition 3.7 and the fact that $T_{k}(u)=u^{+} \wedge k-u^{-}$ $\wedge k$.

Now we are going to show that any integral solution to (1.1) is the socalled very weak solution to (1.1). Set

$$
F:=\left\{\eta \in D(A):-A \eta \in L^{\infty,+}(E ; m)\right\} .
$$

In the definition of very weak solution we require that test functions are defined in each point of $E$ and not only $m$-a.e. or q.e. In many cases one would take $F \cap C_{b}(E)$. However, in general, it may happen that $D(A) \cap C_{b}(E)=\{0\}$. Therefore as test functions we take excessive $m$-versions of elements of $F$. Such versions are finely-continuous, so are defined everywhere. That each $\eta \in F$ have an excessive $m$-version $\check{\eta}$ follows form [4, Theorem 2.2.1].

Definition 3.9. We say that $u \in L^{1}(E ; m)$ is a very weak solution to (1.1) if

$$
\langle u,-A \eta\rangle=\langle\mu, \check{\eta}\rangle, \quad \eta \in F-F .
$$

Lemma 3.10. Let $\eta \in D(A)$ be a bounded excessive function. Then

$$
R(-A \eta)=\eta \text {. }
$$

Proof. Since $\eta \in E x c,-A \eta \geq 0$. By the very definition of the operator $R$,

$$
R(-A \eta)=\sup _{\alpha>0} R_{\alpha}(-A \eta)=\lim _{\alpha \searrow 0} R_{\alpha}(-A \eta) .
$$

By the resolvent identity,

$$
R_{\alpha}(-A \eta)=\lim _{\beta \rightarrow \infty} \beta R_{\alpha}\left(\eta-\beta R_{\beta} \eta\right)=\lim _{\beta \rightarrow \infty}\left(\beta R_{\beta} \eta-\alpha \beta R_{\beta} R_{\alpha} \eta\right)
$$

Since $\eta$ is bounded and excessive,

$$
\lim _{\beta \rightarrow \infty}\left(\beta R_{\beta} \eta-\alpha \beta R_{\beta} R_{\alpha} \eta\right)=\eta-\alpha R_{\alpha} \eta
$$

so $R_{\alpha}(-A \eta)=\eta-\alpha R_{\alpha} \eta$. Since $\eta$ is bounded and excessive and $\eta \in D(A)$, there exists $\xi \in \mathcal{B}^{+}(E)$ such that $\eta=R \xi$. Hence $\alpha R_{\alpha} \eta=\alpha R_{\alpha} R \xi=R \xi-R_{\alpha} \xi \rightarrow 0$ as $\alpha \searrow 0$. Thus $\lim _{\alpha \searrow 0} R_{\alpha}(-A \eta)=\eta$, and the proof is complete.

Proposition 3.11. Let $\mu \in \mathcal{M}_{b}(E)$ and $u \in L^{1}(E ; m)$ be an integral solution to (1.1). Then $u$ is a very weak solution to (1.1).

Proof. Let $\eta \in F$. By the remark preceding Definition 3.9, there exists an $m$-version $\check{\eta}$ of $\eta$ such that $\check{\eta}$ is excessive. Applying Lemma 3.10 we have

$$
\langle u,-A \eta\rangle=\langle R \mu,-A \check{\eta}\rangle=\langle\mu, R(-A \check{\eta})\rangle=\langle\mu, \check{\eta}\rangle,
$$

which proves the proposition. 


\section{Renormalized solutions for general measure data}

In this section, we consider two equivalent definitions of renormalized solution to (1.1) and we study their relations to other concepts of solutions considered in Sect. 3. The first definition was introduced in [10]. The second is new. Its advantage over the first one is that it is simpler because it does not involve the notion of the potential of measure.

\subsection{First definition}

Definition 4.1. Let $\mu \in \mathcal{M}_{b}(E)$. A Borel measurable function $u$ on $E$ is called a renormalized solution to (1.1) if

(i) $T_{k}(u) \in D_{e}(\mathcal{E})$ for every $k>0$,

(ii) there exists a family $\left(\nu_{k}\right)_{k>0} \subset \mathcal{M}_{0, b}(E)$ such that

$$
\mathcal{E}\left(T_{k}(u), \eta\right)=\left\langle\mu_{d}, \eta\right\rangle+\left\langle\nu_{k}, \eta\right\rangle, \quad \eta \in D_{e}(\mathcal{E}) \cap \mathcal{B}_{b}(E), k \geq 0,
$$

(iii) $\lim _{k \rightarrow \infty} R \nu_{k} \rightarrow R \mu_{c} m$-a.e.

Remark 4.2. In [8] it is shown that if $\mu \in \mathcal{M}_{0, b}(E)$ and $u$ is a renormalized solution to (1.1), then $\left\|\nu_{k}\right\|_{T V} \rightarrow 0$ as $k \rightarrow \infty$, where $\left\|\nu_{k}\right\|_{T V}=\left|\nu_{k}\right|(E)$.

By [10, Theorem 4.4], each renormalized solution to (1.1) has an $m$ version which is quasi-continuous. From now on we always consider quasicontinuous versions of renormalized solutions to (1.1).

Proposition 4.3. Let $\mu \in \mathcal{M}_{b}(E)$. Then

(i) $u$ is a renormalized solution to (1.1) if and only if it is an integral solution to $(1.1)$.

(ii) There exists at most one renormalized solution to (1.1).

Proof. Follows from Proposition 3.6 and [10, Theorem 4.4].

Our goal is to show that condition (iii) in Definition 4.1 may be replaced by the following condition (iii'): $\nu_{k} \rightarrow \mu_{c}$ in the narrow topology.

Proposition 4.4. Let $\mu \in \mathcal{M}_{b}(E)$ and $u$ be a renormalized solution to (1.1). Then for every bounded excessive function $\eta$,

$$
\lim _{k \rightarrow \infty}\left\langle\frac{1}{2} \lambda_{k}(u), \eta\right\rangle=\left\langle\mu_{c}^{+}, \eta\right\rangle, \quad \lim _{k \rightarrow \infty}\left\langle\frac{1}{2} \lambda_{-k}(u), \eta\right\rangle=\left\langle\mu_{c}^{-}, \eta\right\rangle .
$$

Proof. We will prove the first assertion. The proof of the second one is analogous. Write $\nu_{k}^{1}:=-\mathbf{1}_{\{u>k\}} \cdot \mu_{d}+\frac{1}{2} \lambda_{k}(u)-\frac{1}{2} \lambda_{0}(u)$. By Proposition 3.7,

$$
-A\left(u^{+} \wedge k\right)=\mathbf{1}_{\{u>0\}} \cdot \mu_{d}+\nu_{k}^{1} \text {. }
$$

Let $\eta$ be a bounded excessive function. From the above equation, Proposition 4.3 and Revuz duality we conclude that

$$
\begin{aligned}
\left\langle\nu_{k}^{1}, \eta\right\rangle & =\lim _{t \rightarrow 0^{+}} \frac{1}{t} E_{\eta \cdot m} \int_{0}^{t} d A_{r}^{\nu_{k}^{1}}=\lim _{t \rightarrow 0^{+}} \frac{1}{t}\left\langle R \nu_{k}^{1}-P_{t} R \nu_{k}^{1}, \eta\right\rangle \\
& =\lim _{t \rightarrow 0^{+}}\left[-\frac{1}{t}\left\langle R\left(\mathbf{1}_{\{u>0\}} \cdot \mu_{d}\right)-P_{t} R\left(\mathbf{1}_{\{u>0\}} \cdot \mu_{d}\right), \eta\right\rangle\right.
\end{aligned}
$$




$$
\begin{aligned}
& \left.+\frac{1}{t}\left\langle u^{+} \wedge k-P_{t}\left(u^{+} \wedge k\right), \eta\right\rangle\right] \\
= & \lim _{t \rightarrow 0^{+}}\left[-\frac{1}{t} E_{\eta \cdot m} \int_{0}^{t} d A_{r}^{\mathbf{1}_{\{u>0\}} \cdot \mu_{d}}+\frac{1}{t}\left\langle u^{+} \wedge k, \eta-P_{t} \eta\right\rangle\right] \\
= & -\left\langle\mathbf{1}_{\{u>0\}} \cdot \mu_{d}, \eta\right\rangle+\lim _{t \rightarrow 0^{+}} \frac{1}{t}\left\langle u^{+} \wedge k, \eta-P_{t} \eta\right\rangle .
\end{aligned}
$$

Since $\eta \in E x c$, we deduce from the above equation that $\left\langle\nu_{k}^{1}, \eta\right\rangle$ is nondecreasing. Therefore we may pass to the limit in the above equation as $k \rightarrow \infty$. We then have

$$
\begin{aligned}
& \lim _{k \rightarrow \infty} \lim _{t \rightarrow 0^{+}} \frac{1}{t}\left\langle u^{+} \wedge k, \eta-P_{t} \eta\right\rangle \\
& =\lim _{k \rightarrow \infty} \lim _{t \rightarrow 0^{+}} \frac{1}{t}\left\langle u^{+} \wedge k+R\left(\mathbf{1}_{\{0<u \leq k\}} \cdot \mu_{d}^{-}\right)+\frac{1}{2} R\left(\lambda_{0}(u)\right), \eta-P_{t} \eta\right\rangle \\
& \quad-\lim _{k \rightarrow \infty} \lim _{t \rightarrow 0^{+}} \frac{1}{t}\left\langle R\left(\mathbf{1}_{\{0<u \leq k\}} \cdot \mu_{d}^{-}\right)+\frac{1}{2} R\left(\lambda_{0}(u)\right), \eta-P_{t} \eta\right\rangle .
\end{aligned}
$$

It is clear that $R\left(\mathbf{1}_{\{0<u \leq k\}} \cdot \mu_{d}^{-}\right)+\frac{1}{2} R\left(\lambda_{0}(u)\right)$ is an excessive function. By (3.7), $u^{+} \wedge k+R\left(\mathbf{1}_{\{0<u \leq k\}} \cdot \mu_{d}^{-}\right)+\frac{1}{2} R\left(\lambda_{0}(u)\right)$ is also an excessive function. Therefore both limits with respect to $t$ on the right-hand side of (4.1) are nondecreasing. It is clear that this is also true for limits with respect to $k$. Therefore we may change the order of the limits in (4.1). By Proposition 3.7, we then have

$$
\begin{aligned}
\lim _{k \rightarrow \infty} \lim _{t \rightarrow 0^{+}} \frac{1}{t}\left\langle u^{+} \wedge k, \eta-P_{t} \eta\right\rangle & =\lim _{t \rightarrow 0^{+}} \lim _{k \rightarrow \infty} \frac{1}{t}\left\langle u^{+} \wedge k, \eta-P_{t} \eta\right\rangle \\
& =\lim _{t \rightarrow 0^{+}} \frac{1}{t}\left\langle u^{+}, \eta-P_{t} \eta\right\rangle \\
& =\lim _{t \rightarrow 0^{+}} \frac{1}{t}\left\langle u^{+}-P_{t} u^{+}, \eta\right\rangle \\
& =\left\langle\mathbf{1}_{\{u>0\}} \cdot \mu_{d}-\frac{1}{2} \lambda_{0}(u)+\mu_{c}^{+}, \eta\right\rangle .
\end{aligned}
$$

This proves the first assertion.

Lemma 4.5. Let $h \in C_{b}(E)$. Then there exist sequences $\left\{h_{n}^{1}\right\},\left\{h_{n}^{2}\right\} \in E x c-E x c$ such that

(i) $-\|h\|_{\infty} \leq h_{n}^{2} \leq h \leq h_{n}^{1} \leq\|h\|_{\infty}, n \geq 1$,

(ii) $h_{n}^{2} \nearrow h, h_{n}^{1} \searrow h$ as $n \rightarrow \infty$.

Proof. Since $(\mathcal{E}, D(\mathcal{E}))$ is transient, there exists a strictly positive bounded function $g_{0}$ such that $R g_{0}$ is bounded (see [12, Corollary 1.3.6.]). Set $g:=R_{1} g_{0}$. Then $R g=R R_{1} g_{0}=R R_{1} R g_{0} \leq R g_{0}=g$. It is clear that $g$ is bounded, finelycontinuous and strictly positive. For $n \geq 1$, we set

$$
\begin{array}{r}
h_{n}^{1}(x)=\sup _{\tau \in \mathcal{T}} E_{x}\left(-n \int_{0}^{\tau} g\left(X_{r}\right) d r+h\left(X_{\tau}\right)\right), \\
h_{n}^{2}(x)=\inf _{\tau \in \mathcal{T}} E_{x}\left(n \int_{0}^{\tau} g\left(X_{r}\right) d r+h\left(X_{\tau}\right)\right),
\end{array}
$$


where $\mathcal{T}$ is the set of all $\mathbb{F}$-stopping times. We have

$$
h_{n}^{2}(x)=-\sup _{\tau \in \mathcal{T}} E_{x}\left(-n \int_{0}^{\tau} g\left(X_{r}\right) d r+\left(-h\left(X_{\tau}\right)\right)\right),
$$

so $-h_{n}^{2}$ is defined as $h_{n}^{1}$ but with $h$ replaced by $-h$. Therefore it is enough to prove that $\left\{h_{n}^{1}\right\}$ has the desired properties. Observe that

$$
h_{n}^{1}(x)+n R g(x)=\sup _{\tau \in \mathcal{T}} E_{x}\left((n R g+h)\left(X_{\tau}\right)\right)
$$

Hence, by [11], $h_{n}^{1}+n R g$ is an excessive function. It is clear that $n R g$ is also excessive. Thus $h_{n}^{1} \in$ Exc - Exc. By the definition, $\left\{h_{n}^{1}\right\}$ is nonincreasing. Moreover, with $\tau_{0}=0$, we have

$$
\begin{aligned}
h(x) & =E_{x}\left(-n \int_{0}^{\tau_{0}} g\left(X_{r}\right) d r+h\left(X_{\tau_{0}}\right)\right) \\
& \leq h_{n}^{1}(x)=\sup _{\tau \in \mathcal{T}} E_{x}\left(-n \int_{0}^{\tau} g\left(X_{r}\right) d r+h\left(X_{\tau}\right)\right) \leq\|h\|_{\infty} .
\end{aligned}
$$

Since $R g$ is an excessive function and $h$ is continuous, the process $(n R g+h)(X)$ is càdlàg under the measure $P_{x}$ for every $x \in E$. For every $\varepsilon>0$ there exists $\tau_{n, \varepsilon}^{x} \in \mathcal{T}$ such that

$$
\begin{aligned}
& E_{x}\left(-n \int_{0}^{\tau_{n, \varepsilon}^{x}} g\left(X_{r}\right) d r+h\left(X_{\tau_{n, \varepsilon}^{x}}\right)\right)-\varepsilon \\
& \quad \leq h_{n}^{1}(x) \leq E_{x}\left(-n \int_{0}^{\tau_{n, \varepsilon}^{x}} g\left(X_{r}\right) d r+h\left(X_{\tau_{n, \varepsilon}^{x}}\right)\right)+\varepsilon .
\end{aligned}
$$

From this we conclude that

$$
n E_{x} \int_{0}^{\tau_{n, \varepsilon}^{x}} g\left(X_{r}\right) d r \leq 2\|h\|_{\infty}+\varepsilon .
$$

Assume for a moment that we know that the above inequality implies that $\tau_{n, \varepsilon}^{x} \rightarrow 0$ in probability $P_{x}$ as $n \rightarrow \infty$. Then, by continuity of $h$ and (4.2),

$$
\begin{aligned}
& h_{n}^{1}(x) \leq E_{x}\left(-n \int_{0}^{\tau_{n, \varepsilon}^{x}} g\left(X_{r}\right) d r+h\left(X_{\tau_{n, \varepsilon}^{x}}\right)\right)+\varepsilon \\
& \quad \leq E_{x}\left(h\left(X_{\tau_{n, \varepsilon}^{x}}\right)\right)+\varepsilon \rightarrow h(x)+\varepsilon .
\end{aligned}
$$

This implies that $\lim _{n \rightarrow \infty} h_{n}^{1}(x) \leq h(x)$. Since, $h_{n}^{1} \geq h$, we get the desired result. What is left is to show that $\tau_{n, \varepsilon}^{x} \rightarrow 0$ in probability $P_{x}$ as $n \rightarrow \infty$. Aiming for a contradiction, suppose that there exist $\varepsilon_{1}, \varepsilon_{2}>0$ and a subsequence [still denoted by $(n)$ ] such that

$$
P_{x}\left(\tau_{n, \varepsilon}^{x}>\varepsilon_{1}\right)>\varepsilon_{2}, \quad n \geq 1 .
$$

Since $g$ is strictly positive, there exists $\delta>0$ such that $g(x) \geq 2 \delta$. Set

$$
\sigma^{x}=\inf \left\{t>0:\left|g\left(X_{t}\right)-g(x)\right|>\delta\right\} .
$$


Since $g$ is finely-continuous, for any sequence $t_{n} \searrow 0, \lim _{n \rightarrow \infty} P_{x}\left(\sigma^{x}>t_{n}\right)=1$. Let $t_{n_{0}}>0$ be such that $P_{x}\left(\sigma^{x}>t_{n_{0}}\right) \geq 1-\frac{\varepsilon_{2}}{2}$. Then $P_{x}\left(\tau_{n, \varepsilon}^{x}>\varepsilon_{1}, \sigma^{x}>\right.$ $\left.t_{n_{0}}\right) \geq \varepsilon_{2} / 2$. Hence

$$
\begin{aligned}
E_{x} \int_{0}^{\tau_{n, \varepsilon}^{x}} g\left(X_{r}\right) d r & \geq E_{x} \mathbf{1}_{\left\{\tau_{n, \varepsilon}^{x}>\varepsilon_{1}\right\}} \int_{0}^{\varepsilon_{1} \wedge \sigma^{x}} g\left(X_{r}\right) d r \geq(g(x)-\delta) E_{x} \mathbf{1}_{\left\{\tau_{n, \varepsilon}^{x}>\varepsilon_{1}\right\}} \varepsilon \wedge \sigma^{x} \\
& \geq \varepsilon_{1} \wedge t_{n_{0}}(g(x)-\delta) P_{x}\left(\tau_{n, \varepsilon}^{x}>\varepsilon_{1}, \sigma^{x}>t_{n_{0}}\right) \\
& \geq \frac{\varepsilon_{2}}{2}(g(x)-\delta) \varepsilon_{1} \wedge t_{n_{0}}
\end{aligned}
$$

in contradiction with (4.3).

Theorem 4.6. Let $u$ be a renormalized solution to (1.1). Then

$$
\frac{1}{2} \lambda_{-k}(u) \rightarrow \mu_{c}^{-}, \quad \frac{1}{2} \lambda_{k}(u) \rightarrow \mu_{c}^{+}
$$

as $k \rightarrow \infty$ in the narrow topology.

Proof. By Proposition 4.4,

$$
\frac{1}{2}\left\langle\lambda_{k}(u), \eta\right\rangle \rightarrow\left\langle\mu_{c}^{+}, \eta\right\rangle
$$

for every bounded $\eta \in$ Exc. In particular, $\sup _{k>1}\left\langle\lambda_{k}(u), 1\right\rangle<\infty$. Therefore, there exists a subsequence (still denoted by $(k))$ and a positive $\nu^{1} \in \mathcal{M}_{b}(E)$ such that $\frac{1}{2} \lambda_{k}(u) \rightarrow \nu^{1}$ in the vague topology. Let $h \in C_{c}(E)$. Let $\left\{h_{n}^{1}\right\},\left\{h_{n}^{2}\right\}$ be sequences satisfying properties asserted in Lemma 4.5. Then

$$
\left\langle\mu_{c}^{+}, h_{n}^{2}\right\rangle=\lim _{k \rightarrow \infty}\left\langle\lambda_{k}(u), h_{n}^{2}\right\rangle \leq \lim _{k \rightarrow \infty}\left\langle\lambda_{k}(u), h\right\rangle=\left\langle\nu^{1}, h\right\rangle
$$

and

$$
\left\langle\nu^{1}, h\right\rangle=\lim _{k \rightarrow \infty}\left\langle\lambda_{k}(u), h\right\rangle \leq \lim _{k \rightarrow \infty}\left\langle\lambda_{k}(u), h_{n}^{1}\right\rangle=\left\langle\mu_{c}^{+}, h_{n}^{1}\right\rangle .
$$

Consequently, passing to the limit with $n \rightarrow \infty$ yields $\nu^{1}=\mu_{c}^{+}$. Thus $\frac{1}{2} \lambda_{k}(u) \rightarrow \mu_{c}^{+}$in the vague topology. Since 1 is an excessive function, we also have $\left\langle\frac{1}{2} \lambda_{k}(u), 1\right\rangle \rightarrow\left\langle\mu_{c}^{+}, 1\right\rangle$, so $\frac{1}{2} \lambda_{k}(u) \rightarrow \mu_{c}^{+}$in the narrow topology. The proof of the second convergence is similar, so we omit it.

Corollary 4.7. Let $u$ be a renormalized solution to (1.1). Then

$$
\nu_{k} \rightarrow \mu_{c}, \quad\left|\nu_{k}\right| \rightarrow\left|\mu_{c}\right|
$$

as $k \rightarrow \infty$ in the narrow topology.

Proof. By Proposition 4.3 and Remark 3.2, $u$ is quasi-continuous. Therefore $\mathbf{1}_{\{-k<u \leq k\}} \cdot \mu_{d} \rightarrow 0$ in the total variation norm. Hence, by Corollary 3.8 and Theorem 4.6, $\nu_{k} \rightarrow \mu_{c}$ in the narrow topology. By [2, Theorem 8.4.7],

$$
\liminf _{k \rightarrow \infty}\left|\nu_{k}\right|(E) \geq\left|\mu_{c}\right|(E) \text {. }
$$

On the other hand, using Corollary 3.8, Theorem 4.6 and the fact that $\mathbf{1}_{\{-k<u \leq k\}} \cdot \mu_{d} \rightarrow 0$ in the total variation norm, we get

$$
\limsup _{k \rightarrow \infty}\left|\nu_{k}\right|(E) \leq \limsup _{k \rightarrow \infty} \frac{1}{2} \lambda_{k}(u)(E)+\limsup _{k \rightarrow \infty} \frac{1}{2} \lambda_{-k}(u)(E)=\left|\mu_{c}\right|(E) .
$$


Thus $\lim _{k \rightarrow \infty}\left|\nu_{k}\right|(E)=\left|\mu_{c}\right|(E)$. From this and [2, Theorem 8.4.7] we get the desired result.

\subsection{Second definition}

Definition 4.8. Let $\mu \in \mathcal{M}_{b}(E)$. We say that $u \in \mathcal{B}(E)$ is a renormalized solution to (1.1) if

(i) $T_{k}(u) \in D_{e}(\mathcal{E})$ for every $k \geq 0$,

(ii) there exists a family $\left(\nu_{k}\right)_{k>0} \subset \mathcal{M}_{0, b}(E)$ such that

$$
\mathcal{E}\left(T_{k}(u), \eta\right)=\left\langle\mu_{d}, \eta\right\rangle+\left\langle\nu_{k}, \eta\right\rangle, \quad \eta \in D_{e}(\mathcal{E}) \cap \mathcal{B}_{b}(E), k \geq 0,
$$

(iii) $\nu_{k} \rightarrow \mu_{c}$ in the narrow topology.

Theorem 4.9. Let $\mu \in \mathcal{M}_{b}(E)$.

(i) If $u$ is a renormalized solution to (1.1) in the sense of Definition 4.1, then $u$ is a renormalized solution to (1.1) in the sense of Definition 4.8.

(ii) Assume that either $R_{\alpha}\left(C_{b}(E)\right) \subset C_{b}(E)$ for some (hence for all) $\alpha>0$ and $u \in L^{1}(E ; m)$ or $R_{\alpha}\left(\mathcal{B}_{b}(E)\right) \subset C_{b}(E)$ for some (hence for all) $\alpha>0$. If $u$ is a renormalized solution to (1.1) in the sense of Definition 4.8, then $u$ is a renormalized solution to (1.1) in the sense of Definition 4.1.

Proof. Assertion (i) follows from Proposition 4.3, Corollary 3.8 and Theorem 4.6 (since $u$ is quasi-continuous it is finite q.e.). To prove (ii), assume that $u$ is a renormalized solution to (1.1) in the sense of Definition 4.8. By Definition 4.8(ii),

$$
T_{k}(u)=R \mu_{d}+R \nu_{k}, \quad k \geq 0, \quad \text { q.e. }
$$

Therefore $\left\{R \nu_{k}\right\}$ converges q.e. as $k \rightarrow \infty$. Let $v$ denote its limit. Let $\eta \in \mathcal{B}(E)$ be a bounded positive function such that $R \eta$ is bounded. Observe that

$$
\langle|u|, \eta\rangle=\sup _{k \geq 1}\left\langle T_{k}(u), \operatorname{sign}(u) \eta\right\rangle \leq\left\langle\left|\mu_{d}\right|, R \eta\right\rangle+\sup _{k \geq 1}\left\langle\left|\nu_{k}\right|, R \eta\right\rangle .
$$

Since $\nu_{k}$ is narrowly convergent, $\sup _{k \geq 1}\left\langle\left|\nu_{k}\right|, \eta\right\rangle<\infty$. Therefore $\langle|u|, \eta\rangle<\infty$ for every $\eta \in \mathcal{B}^{+}(E)$ such that $R \eta$ is bounded. We also have

$$
\left|R \nu_{k}\right| \leq R\left|\mu_{d}\right|+|u| \text {. }
$$

Assume that $R_{\alpha}\left(\mathcal{B}_{b}(E)\right) \subset C_{b}(E)$ for every $\alpha>0$, i.e. $\left(R_{\alpha}\right)_{\alpha>0}$ has the strong Feller property. Then, by (4.5) and the Lebesgue dominated convergence theorem,

$$
\begin{aligned}
& \left\langle v, \alpha R_{\alpha} \eta\right\rangle=\lim _{k \rightarrow \infty}\left\langle R \nu_{k}, \alpha R_{\alpha} \eta\right\rangle=\lim _{k \rightarrow \infty}\left\langle\nu_{k}, \alpha R_{\alpha} R \eta\right\rangle \\
& =\left\langle\mu_{c}, \alpha R_{\alpha} R \eta\right\rangle=\left\langle R \mu_{c}, \alpha R_{\alpha} \eta\right\rangle .
\end{aligned}
$$

The third equation follows from strong Feller property. Letting $\alpha \rightarrow \infty$ gives

$$
\langle v, \eta\rangle=\left\langle R \mu_{c}, \eta\right\rangle \text {. }
$$

Since $\eta \in \mathcal{B}(E)$ was an arbitrary positive function such that $R \eta$ is bounded, $v=R \mu_{c}$. Therefore, by (4.4),

$$
u=R \mu_{d}+R \mu_{c}=R \mu, \quad \text { q.e., }
$$


so by Proposition 4.3, $u$ is a renormalized solution to (1.1) in the sense of Definition 4.1. Assume now that $R_{\alpha}\left(C_{b}(E)\right) \subset C_{b}(E)$ for every $\alpha>0$. For every $\eta \in C_{b}(E)$ we have

$$
\left\langle T_{k}(u), \eta\right\rangle=\left\langle\alpha R_{\alpha} T_{k}(u), \eta\right\rangle+\left\langle\mu_{d}, R_{\alpha} \eta\right\rangle+\left\langle\nu_{k}, R_{\alpha} \eta\right\rangle .
$$

Letting $k \rightarrow \infty$ and then $\alpha \searrow 0$ shows that $u=R \mu$. By Proposition 4.3 again, $u$ is a renormalized solution to (1.1) in the sense of Definition 4.1.

Corollary 4.10. Let $\mu \in \mathcal{M}_{b}(E)$ and the assumptions of Theorem 4.9(ii) hold. Then there exists a unique renormalized solution $u$ to (1.1) in the sense of Definition 4.8. Moreover,

$$
u(x)=\int_{E} G(x, y) \mu(d y), \quad m \text {-a.e. } x \in E .
$$

Remark 4.11. Even in the case of local operators Definition 4.8 of renormalized solutions to (1.1) is in some cases more convenient in applications then the other definitions considered in [3]. For instance, Petitta et al. [14] applied formulation of this type to solve evolution equations with smooth measure data and absorption on the right-hand side.

Let $f: E \times \mathbb{R} \rightarrow \mathbb{R}$ be a measurable function. In [10] we have proved a uniqueness result for solutions, in the sense of Definition 4.1, to semilinear equations (1.12). Thanks to the equivalence proved in Theorem 4.9 we have the uniqueness result for solutions to (1.12) in the sense of Definition 4.8. Let us also note here that the existence of renormalized solutions to semilinear equations (1.12) with smooth measure data and $f$ satisfying merely the sign condition with respect to the second variable was proved in [9]. In the case of general measure data the existence problem for (1.12) is a very subtle matter. Its investigation requires introducing the notion of reduced measures (see [6]).

Definition 4.12. Let $\mu \in \mathcal{M}_{b}(E)$. We say that $u \in \mathcal{B}(E)$ is a renormalized solution to (1.12) if

(i) $T_{k}(u) \in D_{e}(\mathcal{E})$ for every $k \geq 0$, and $f(\cdot, u) \in L^{1}(E ; m)$,

(ii) there exists a family $\left(\nu_{k}\right)_{k>0} \subset \mathcal{M}_{0, b}(E)$ such that

$$
\mathcal{E}\left(T_{k}(u), \eta\right)=\langle f(\cdot, u), \eta\rangle+\left\langle\mu_{d}, \eta\right\rangle+\left\langle\nu_{k}, \eta\right\rangle, \quad \eta \in D_{e}(\mathcal{E}) \cap \mathcal{B}_{b}(E), k \geq 0,
$$

(iii) $\nu_{k} \rightarrow \mu_{c}$ in the narrow topology.

Theorem 4.13. Let $\mu \in \mathcal{M}_{b}(E)$ and $f$ be non-increasing with respect to the second variable.

(i) If $R_{\alpha}\left(C_{b}(E)\right) \subset C_{b}(E)$ for some $\alpha>0$, then there exists at most one renormalized solution $u \in L^{1}(E ; m)$ to (1.12) in the sense of Definition 4.12 .

(ii) If $R_{\alpha}\left(\mathcal{B}_{b}(E)\right) \subset C_{b}(E)$ for some $\alpha>0$, then there exists at most one renormalized solution to (1.12) in the sense of Definition 4.12.

Proof. Follows from Theorem 4.9, [10, Theorem 4.4] and [6, Corollary 4.3]. 


\section{Structure of renormalized solutions}

From now on by saying renormalized solution we mean a renormalized solution in the sense of Definition 4.1. Recall that by the definition of renormalized solution to (1.1) we have

$$
-A T_{k}(u)=\mu_{d}+\nu_{k},
$$

where $\nu_{k}$ is a bounded smooth measure. By Corollary 3.8,

$$
\nu_{k}=-\mathbf{1}_{\{u>k, u \leq-k\}} \mu_{d}+\frac{1}{2}\left(\lambda_{k}(u)-\lambda_{-k}(u)\right),
$$

and by the definition of the measure $\lambda_{k}(u)$ [see (2.4)],

$$
\lambda_{k}(u)=l_{k}(u)+j_{k}(u) .
$$

In this section, we study the structure of the measures $l_{k}(u)$ and $j_{k}(u)$. We show that $j_{k}(u)$ has an explicit formula via $u$ and the kernel of the nonlocal part of the operator $A$. As for the measure $l_{k}(u)$, we show the so-called reconstruction formula which is well known for equations with measure data and local (nonlinear) Leray-Lions type operators (see, e.g., [3]).

We adopt the notation introduced in Introduction. For the BeurlingDeny decomposition of $(\mathcal{E}, D(\mathcal{E}))$ we defer the reader to [4]. Note that for every $u \in D_{e}(\mathcal{E})$ there exists a unique smooth Radon measure $\mu_{\langle u\rangle}^{c}$ such that

$$
\left\langle\mu_{\langle u\rangle}^{c}, \eta\right\rangle=2 \mathcal{E}^{(c)}(u \eta, u)-\mathcal{E}^{(c)}\left(u^{2}, \eta\right), \quad \eta \in C_{c}(E) \cap D(\mathcal{E})
$$

(see $[4,(3.2 .20)])$ By $[4$, Lemma 5.3.3],

$$
\mu_{\langle u\rangle}^{c}=\mathcal{R}\left([u(X)]^{c}\right), \quad u \in D_{e}(\mathcal{E}),
$$

where $[u(X)]^{c}$ is the continuous part of the square bracket of the semimartingale $u(X)$.

By the probabilistic definition of a solution to (1.1), $u(X)$ is a semimartingale. Therefore $[u(X)]^{c}$ and hence $\mathcal{R}\left([u(X)]^{c}\right)$ are well defined also for solutions to $(1.1)$, although in general, solutions are not in $D_{e}(\mathcal{E})$. In this case, we set $\mu_{\langle u\rangle}^{c}=\mathcal{R}\left([u(X)]^{c}\right)$. By the definition of a renormalized solution to (1.1), $T_{k}(u) \in D_{e}(\mathcal{E})$. By [4, Lemma 5.6.4],

$$
\mathbf{1}_{\{-k<u \leq k\}} \mu_{\langle u\rangle}^{c}=\mu_{\left\langle T_{k}(u)\right\rangle}^{c} .
$$

Moreover, by [4, Lemma 3.2.3], $\mu_{\left\langle T_{k}(u)\right\rangle}^{c}$ is bounded. On the other hand, by (5.1), for every $\eta \in D_{e}(\mathcal{E}) \cap \mathcal{B}_{b}(E)$ we have

$$
\left\langle\mu_{\left\langle T_{k}(u)\right\rangle}^{c}, \eta\right\rangle=2 \mathcal{E}^{(c)}\left(T_{k}(u) \eta, T_{k}(u)\right)-\mathcal{E}^{(c)}\left(T_{k}(u)^{2}, \eta\right) .
$$

By this and (5.3),

$$
\left\langle\mu_{\langle u\rangle}^{c}, \eta\right\rangle=\lim _{k \rightarrow \infty} 2 \mathcal{E}^{(c)}\left(T_{k}(u) \eta, T_{k}(u)\right)-\mathcal{E}^{(c)}\left(T_{k}(u)^{2}, \eta\right), \quad \eta \in D_{e}(\mathcal{E}) \cap \mathcal{B}_{b}(E) .
$$

In general, $\mu_{\langle u\rangle}^{c}$ is not a Radon measure. However, by (5.3) and the fact that $\mu_{\left\langle T_{k}(u)\right\rangle}^{c}$ is bounded, $\left\langle\mu_{\langle u\rangle}^{c},|h(u) \eta|\right\rangle$ is finite for all $\eta \in \mathcal{B}_{b}(E)$ and $h \in \mathcal{B}_{b}(E)$ such that $h$ has compact support. 
Proposition 5.1. Let $\mu \in \mathcal{M}_{b}(E)$ and $u$ be a renormalized solution to (1.1). Then for every $k>0$,

$$
\begin{aligned}
j_{k}(u)(d x)= & 2 \int_{E}(|u(y)-k|-|u(x)-k|-\operatorname{sign}(u(x)-k)(u(y)-u(x))) J(d x, d y) \\
& +\left(\mathbf{1}_{\{u(x)>k\}}(|k|+k)+\mathbf{1}_{\{u(x) \leq k\}}(|k|-k)\right) \kappa(d x) .
\end{aligned}
$$

Proof. Let $k>0$ and $(N, H)$ be a Lévy system of $\mathbb{X}$. By [4, (A.3.23)],

$$
\begin{aligned}
p\left[J^{k}(u(X))\right]_{t}= & \int_{0}^{t} \int_{E \cup\{\partial\}}\left(|u(y)-k|-\left|u\left(X_{s}\right)-k\right|\right. \\
& \left.-\operatorname{sign}\left(u\left(X_{s}\right)-k\right)\left(u(y)-u\left(X_{s}\right)\right)\right) N\left(X_{s}, d y\right) d H_{s} .
\end{aligned}
$$

Put $\beta=\mathcal{R}(H)$. From the above equation it follows that

$$
\begin{aligned}
& j_{k}(u)(d x) \\
& =\int_{E \cup\{\partial\}}(|u(y)-k|-|u(x)-k|-\operatorname{sign}(u(x)-k)(u(y)-u(x))) N(x, d y) \beta(d x) .
\end{aligned}
$$

Hence

$$
\begin{aligned}
j_{k}(u)(d x)= & \int_{E}(|u(y)-k|-|u(x)-k|-\operatorname{sign}(u(x)-k)(u(y)-u(x))) N(x, d y) \beta(d x) \\
& +(|u(\partial)-k|-|u(x)-k|-\operatorname{sign}(u(x)-k)(u(\partial)-u(x))) N(x,\{\partial\}) \beta(d x) .
\end{aligned}
$$

This implies the desired equality because by convention $u(\partial)=0$, and by [4, Theorem 5.3.1], $N(x,\{\partial\}) \beta(d x)=\kappa(d x)$ and $N(x, d y) \beta(d x)=2 J$ $(d x, d y)$.

Proposition 5.2. Let $\mu \in \mathcal{M}_{b}(E)$ and $u$ be a renormalized solution to (1.1). Then for every bounded $\eta \in$ Exc and every $\varphi \in \mathcal{B}^{+}(E)$,

$$
\int_{\mathbb{R}}\left\langle l_{a}(u), \eta\right\rangle \varphi(a) d a=\left\langle\mu_{\langle u\rangle}^{c}, \varphi(u) \eta\right\rangle .
$$

Proof. By (2.1) and the monotone convergence,

$$
\begin{aligned}
\int_{\mathbb{R}}\left\langle l_{a}(u), \eta\right\rangle \varphi(a) d a & =\int_{\mathbb{R}}\left[\lim _{t \searrow 0} \frac{1}{t} E_{\eta \cdot m} \int_{0}^{t} d L_{r}^{a}(u(X))\right] \varphi(a) d a \\
& =\lim _{t \searrow 0} \frac{1}{t}\left[\int_{\mathbb{R}} E_{\eta \cdot m} \int_{0}^{t} d L_{r}^{a}(u(X))\right] \varphi(a) d a .
\end{aligned}
$$

Applying now the occupation time formula (see [15, Corollary 1, p. 216]) we get

$$
\int_{\mathbb{R}}\left\langle l_{a}(u), \eta\right\rangle \varphi(a) d a=\lim _{t \searrow 0} \frac{1}{t} E_{\eta \cdot m} \int_{0}^{t} \varphi\left(u\left(X_{r}\right)\right) d[u(X)]_{r}^{c},
$$

which by $(2.1)$ and $(5.2)$ is equal to $\left\langle\mu_{\langle u\rangle}^{c}, \varphi(u) \eta\right\rangle$.

Corollary 5.3. Assume that $(\mathcal{E}, D(\mathcal{E}))$ is local (i.e. $J \equiv 0$ in the Beurling-Deny decomposition of $\mathcal{E})$. Let $u$ be a renormalized solution to (1.1). Let $\left\{b_{n}\right\},\left\{c_{n}\right\}$ 
be nondecreasing sequences such that $b_{n}<c_{n}, n \geq 1$ and $b_{n} \nearrow \infty, c_{n} \nearrow \infty$ as $n \rightarrow \infty$. Then for every $\eta \in C_{b}(E)$,

$$
\lim _{n \rightarrow \infty} \frac{1}{c_{n}-b_{n}}\left\langle\mu_{\langle u\rangle}^{c}, \mathbf{1}_{\left\{b_{n} \leq u \leq c_{n}\right\}} \eta\right\rangle=2\left\langle\mu_{c}^{+}, \eta\right\rangle
$$

and

$$
\lim _{n \rightarrow \infty} \frac{1}{c_{n}-b_{n}}\left\langle\mu_{\langle u\rangle}^{c}, \mathbf{1}_{\left\{-c_{n} \leq u \leq-b_{n}\right\}} \eta\right\rangle=2\left\langle\mu_{c}^{-}, \eta\right\rangle .
$$

Proof. Since $J \equiv 0, \lambda_{a}(u)=l_{a}(u)$. Taking now $\varphi=\frac{1}{c_{n}-b_{n}} \mathbf{1}_{\left[b_{n}, c_{n}\right]}$ or $\varphi=$ $\frac{1}{c_{n}-b_{n}} \mathbf{1}_{\left[-c_{n},-b_{n}\right]}$ in (5.4), letting $n \rightarrow \infty$ and using Theorem 4.6 yields the desired convergences.

Example 5.4. Let $D$ be a bounded domain in $\mathbb{R}^{d}$ and $m$ be the Lebesgue measure on $\mathbb{R}^{d}$. Consider the operator

$$
A u=\sum_{i, j=1}^{d}\left(a_{i j} u_{x_{i}}\right)_{x_{j}},
$$

where $a_{i j} \in L_{l o c}^{1}(D ; m)$ and $a=\left[a_{i j}\right]_{i, j=1, \ldots, d}$ is a non-negative definite symmetric matrix. To give a precise definition of the operator $A$, we assume that the form

$$
\mathcal{E}^{0}(u, v):=\int_{D} a \nabla u \nabla v d m, \quad u, v \in C_{c}^{\infty}(D)
$$

is closable. This is satisfied for instance if $a_{i j} \in H_{l o c}^{1}(D)$ for $i, j=1, \ldots, d$ or $a \geq \lambda I$ for some $\lambda>0$ (see, e.g., [4, p. 111]). Let $(\mathcal{E}, D(\mathcal{E}))$ denote the closure of $\left(\mathcal{E}^{0}, C_{c}^{\infty}(D)\right)$. Then there exists a unique self-adjoint operator $(A, D(A))$ such that $D(A) \subset D(\mathcal{E})$, and

$$
\mathcal{E}(u, v)=(-A u, v), \quad u \in D(A), v \in D(\mathcal{E}) .
$$

It is clear that $\mathcal{E}^{(c)}=\mathcal{E}$, so $(\mathcal{E}, D(\mathcal{E}))$ is local. Moreover,

$$
\left\langle\mu_{\langle u\rangle}^{c}, \eta\right\rangle=2 \int_{D} \eta|\sigma \nabla u|^{2} d m,
$$

where $\sigma$ is such that $\sigma \cdot \sigma^{T}=a$. By Corollary 5.3 , for any $\left\{b_{n}\right\},\left\{c_{n}\right\}$ satisfying its assumptions we have

and

$$
\lim _{n \rightarrow \infty} \frac{1}{c_{n}-b_{n}} \int_{\left\{b_{n} \leq u \leq c_{n}\right\}} \eta|\sigma \nabla u|^{2} d m=\left\langle\mu_{c}^{+}, \eta\right\rangle
$$

$$
\lim _{n \rightarrow \infty} \frac{1}{c_{n}-b_{n}} \int_{\left\{-c_{n} \leq u \leq-b_{n}\right\}} \eta|\sigma \nabla u|^{2} d m=\left\langle\mu_{c}^{-}, \eta\right\rangle .
$$

Example 5.5. Let $m$ be the Lebesgue measure on $\mathbb{R}^{d}$ and $\alpha \in(0,1 \wedge(d / 2))$. Consider the fractional Laplace operator $A=\Delta^{\alpha}$ associated with the Dirichlet form on $L^{2}\left(\mathbb{R}^{d} ; m\right)$ defined as

$$
\left\{\begin{array}{l}
\mathcal{E}(u, v)=c(\alpha, d) \int_{\mathbb{R}^{d}} \int_{\mathbb{R}^{d}} \frac{(u(x)-u(y))(v(x)-v(y))}{|x-y|^{d+2 \alpha}} d x d y, \quad u, v \in D(\mathcal{E}), \\
D(\mathcal{E})=\left\{u \in L^{2}\left(\mathbb{R}^{d} ; m\right): \mathcal{E}(u, u)<\infty\right.
\end{array}\right.
$$


where $c(d, \alpha)>0$ is some suitably chosen constant. In this example $\mathcal{E}^{c}=0$, $\kappa=0$ and

$$
J(d x, d y)=\frac{c(\alpha, d)}{|x-y|^{d+2 \alpha}} d x d y,
$$

so by Proposition 5.1,

$$
j_{k}(u)(d x)=c(d, \alpha) \int_{\mathbb{R}^{d}} \frac{|u(y)-k|-|u(x)-k|-\operatorname{sign}(u(x)-k)(u(y)-u(x))}{|x-y|^{d+2 \alpha}} d y d x .
$$

\section{Renormalized solutions for smooth measure data}

In [1] a definition of renormalized solutions to (1.1) with purely jumping operator on $\mathbb{R}^{d}$ and $\mu \in L^{1}\left(\mathbb{R}^{d}\right)$ was introduced. In this section, we show that this definition can be extended to general smooth measure data and the class of operators considered in the present paper, so in particular to the class of operators considered in [5]. We also show that if $\mu \in \mathcal{M}_{0, b}$, then renormalized solutions considered in the previous sections are renormalized solutions in the sense of the new definition formulated below.

Given $h \in C_{c}^{1}(\mathbb{R}), \eta \in D_{e}(\mathcal{E}) \cap \mathcal{B}_{b}(E)$ and $u \in \mathcal{B}(E)$ such that $T_{k}(u) \in$ $D_{e}(\mathcal{E})$ for $k>0$ and $(1.5)$ is satisfied, we set

$$
\begin{aligned}
\mathcal{E}(u, h(u) \eta)= & \mathcal{E}^{(c)}\left(T_{M}(u), h(u) \eta\right) \\
& +\int_{E \times E}(u(x)-u(y))(h(u)(x)-h(u)(y)) \frac{\eta(x)+\eta(y)}{2} J(d x, d y) \\
& +\int_{E \times E}(u(x)-u(y))(\eta(x)-\eta(y)) \frac{h(u)(x)+h(u)(y)}{2} J(d x, d y) \\
& +\int_{E} u(x) h(u)(x) \eta(x) \kappa(d x),
\end{aligned}
$$

where $M>0$ is chosen so that $\operatorname{supp}[h] \subset[-M, M]$. Thanks to the assumptions on $h, \eta$ and $u$ all the integrals appearing in (6.1) are absolutely convergent. Furthermore, by [4, Theorem 3.2.2],

$$
\mathcal{E}^{(c)}\left(T_{M}(u)-T_{M^{\prime}}(u), h(u) \eta\right)=0
$$

for every $M^{\prime}>0$ such that $\operatorname{supp}[h] \subset\left[-M^{\prime}, M^{\prime}\right]$, so $\mathcal{E}(u, h(u) \eta)$ is well defined. Set

$$
\Phi_{k}(u)=T_{k+1}(u)-T_{k}(u), \quad k>0 .
$$

Definition 6.1. Let $\mu \in \mathcal{M}_{0, b}(E)$. We say that $u \in \mathcal{B}(E)$ is a renormalized solution to (1.1) if

(i) $T_{k}(u) \in D_{e}(\mathcal{E})$ for every $k>0$ and (1.5) is satisfied,

(ii) $\mathcal{E}(u, h(u) \eta)=\langle\mu, h(u) \eta\rangle$ for every $\eta \in \mathcal{B}_{b}(E) \cap D_{e}(\mathcal{E})$,

(iii) $\mathcal{E}\left(u, \Phi_{k}(u)\right) \rightarrow 0$ as $k \rightarrow \infty$.

Proposition 6.2. Let $\mu \in \mathcal{M}_{0, b}(E)$. If $u$ is a renormalized solution to (1.1) in the sense of Definition 4.1 then $u$ is a renormalized solution to (1.1) in the sense of Definition 6.1 . 
Proof. By Definition 4.1(ii), for all $k, l>0$,

$$
\mathcal{E}\left(T_{k}(u), T_{l}(u)\right)=\left\langle\mu_{d}, T_{l}(u)\right\rangle+\left\langle\lambda_{k}, T_{l}(u)\right\rangle \leq l\|\mu\|_{T V}+l\left\|\nu_{k}\right\|_{T V} .
$$

Hence

$$
\int_{E \times E}\left(T_{k}(u)(x)-T_{k}(u)(y)\right)\left(T_{l}(u)(x)-T_{l}(u)(y)\right) J(d x, d y) \leq l\|\mu\|_{T V}+l\left\|\nu_{k}\right\|_{T V} .
$$

Letting $k \rightarrow \infty$ and applying Fatou's lemma and Remark 4.2 we get

$$
\int_{E \times E}(u(x)-u(y))\left(T_{l}(u)(x)-T_{l}(u)(y)\right) J(d x, d y) \leq l\|\mu\|_{T V}, \quad l>0 .
$$

From this and condition (i) of Definition 4.1 it follows that $u$ satisfies condition (i) of Definition 6.1. Condition (iii) of Definition 6.1 follows from [7, Proposition 5.10]. As for condition (ii), observe that by condition (ii) of Definition 4.1 and (6.2), for every $k \geq M$ we have

$$
\begin{aligned}
\mathcal{E}^{(c)} & \left(T_{M}(u), h(u) \eta\right) \\
& +\int_{E \times E}\left(T_{k}(u)(x)-T_{k}(u)(y)\right)(h(u)(x)-h(u)(y)) \frac{\eta(x)+\eta(y)}{2} J(d x, d y) \\
& +\int_{E \times E}\left(T_{k}(u)(x)-T_{k}(u)(y)\right)(\eta(x)-\eta(y)) \frac{h(u)(x)+h(u)(y)}{2} J(d x, d y) \\
& +\int_{E} T_{M}(u)(x) h(u)(x) \eta(x) \kappa(d x)=\left\langle\mu_{d}, h(u) \eta\right\rangle+\left\langle\nu_{k}, h(u) \eta\right\rangle .
\end{aligned}
$$

Since $\left|T_{k}(u)(x)-T_{k}(u)(y)\right| \leq|u(x)-u(y)|$, applying the Lebesgue dominated convergence theorem shows that the left-hand side of the above equality tends to $\mathcal{E}(u, h(u) \eta)$ as $k \rightarrow \infty$. On the other hand, by Remark 4.2, $\lim _{k \rightarrow \infty}\left\|\nu_{k}\right\|_{T V}=0$, which shows that condition (ii) of Definition 6.1 is satisfied.

\section{Acknowledgements}

This work was supported by Polish National Science Centre (Grant No. 2017/25/B/ST1/00878).

Open Access. This article is licensed under a Creative Commons Attribution 4.0 International License, which permits use, sharing, adaptation, distribution and reproduction in any medium or format, as long as you give appropriate credit to the original author(s) and the source, provide a link to the Creative Commons licence, and indicate if changes were made. The images or other third party material in this article are included in the article's Creative Commons licence, unless indicated otherwise in a credit line to the material. If material is not included in the article's Creative Commons licence and your intended use is not permitted by statutory regulation or exceeds the permitted use, you will need to obtain permission directly from the copyright holder. To view a copy of this licence, visit http://creativecommons.org/ licenses/by/4.0/.

Publisher's Note Springer Nature remains neutral with regard to jurisdictional claims in published maps and institutional affiliations. 


\section{References}

[1] Alibaud, N., Andreianov, B., Bendahmane, M.: Renormalized solutions of the fractional Laplace equation. C. R. Math. Acad. Sci. Paris 348, 759-762 (2010)

[2] Bogachev, V.I.: Measure Theory, vol. II. Springer, Berlin (2007)

[3] Dal Maso, G., Murat, F., Orsina, L., Prignet, A.: Renormalized solutions of elliptic equations with general measure data. Ann. Scuola Norm. Sup. Pisa Cl. Sci. (4) 28, 741-808 (1999)

[4] Fukushima, M., Oshima, Y., Takeda, M.: Dirichlet Forms and Symmetric Markov Processes, Second revised and extended edn. Walter de Gruyter, Berlin (2011)

[5] Karlsen, K.H., Petitta, F., Ulusoy, S.: A duality approach to the fractional Laplacian with measure data. Publ. Mat. 55, 151-161 (2011)

[6] Klimsiak, T.: Reduced measures for semilinear elliptic equations involving Dirichlet operators. Calc. Var. Partial Differ. Equ. 55 (2016) Art. 78, 27 pp

[7] Klimsiak, T., Rozkosz, A.: Dirichlet forms and semilinear elliptic equations with measure data. J. Funct. Anal. 265, 890-925 (2013)

[8] Klimsiak, T., Rozkosz, A.: Renormalized solutions of semilinear equations involving measure data and operator corresponding to Dirichlet form. NoDEA Nonlinear Differ. Equ. Appl. 22, 1911-1934 (2015)

[9] Klimsiak, T.:, Rozkosz, A.: On semilinear elliptic equations with diffuse measures. NoDEA Nonlinear Differ. Equ. Appl. 25(4) (2018), Art. 35, 23 pp

[10] Klimsiak, T., Rozkosz, A.: Renormalized solutions of semilinear elliptic equations with general measure data. Monatsh. Math. 188, 689-702 (2019)

[11] Nagai, H.: On an optimal stopping problem and a variational inequality. J. Math. Soc. Jpn. 30, 303-312 (1978)

[12] Oshima, Y.: Semi-Dirichlet Forms and Markov Processes. Walter de Gruyter, Berlin (2013)

[13] Petitta, F.: Some remarks on the duality method for integro-differential equations with measure data. Adv. Nonlinear Stud. 16, 115-124 (2016)

[14] Petitta, F., Ponce, A., Porretta, A.: Diffuse measures and nonlinear parabolic equations. J. Evol. Equ. 11, 861-905 (2011)

[15] Protter, P.: Stochastic Integration and Differential Equations, 2nd edn. Springer, Berlin (2004)

[16] Stampacchia, G.: Le problème de Dirichlet pour les èquations elliptiques du second ordre à coefficients discontinus. Ann. Inst. Fourier (Grenoble) 15, 189$258(1965)$ 
Tomasz Klimsiak

Faculty of Mathematics and Computer Science

Nicolaus Copernicus University

Chopina 12/18

87-100 Toruń

Poland

e-mail: tomas@mat.umk.pl

Received: 4 March 2020.

Accepted: 25 July 2020. 